\title{
Solving a Problem of Rotary Motion for a Heavy Solid Using the Large Parameter Method
}

\author{
A. I. Ismail $\mathbb{( i D}^{1,2}$ \\ ${ }^{1}$ Department of Mechanics, College of Engineering and Islamic Architecture, Umm Al-Qura University, P.O. Box 5555, Makkah, \\ Saudi Arabia \\ ${ }^{2}$ Mathematics Department, Faculty of Science, Tanta University, P.O. Box 31527, Tanta, Egypt \\ Correspondence should be addressed to A. I. Ismail; aiismail@uqu.edu.sa
}

Received 29 May 2020; Revised 17 June 2020; Accepted 26 June 2020; Published 1 September 2020

Guest Editor: Abdullah A. Ansari

Copyright (c) 2020 A. I. Ismail. This is an open access article distributed under the Creative Commons Attribution License, which permits unrestricted use, distribution, and reproduction in any medium, provided the original work is properly cited.

\begin{abstract}
The small parameter method was applied for solving many rotational motions of heavy solids, rigid bodies, and gyroscopes for different problems which classify them according to certain initial conditions on moments of inertia and initial angular velocity components. For achieving the small parameter method, the authors have assumed that the initial angular velocity is sufficiently large. In this work, it is assumed that the initial angular velocity is sufficiently small to achieve the large parameter instead of the small one. In this manner, a lot of energy used for making the motion initially is saved. The obtained analytical periodic solutions are represented graphically using a computer program to show the geometric periodicity of the obtained solutions in some interval of time. In the end, the geometric interpretation of the stability of a motion is given.
\end{abstract}

\section{Introduction}

Consider a heavy solid of mass $M$ rotating about a fixed point $O$ in presence of a uniform gravity field of force [1]. The fundamental equations of motion and their three first integrals are presented and reduced to a quasilinear autonomous system having one first integral [2]. Consider that the ellipsoid of inertia of the body is arbitrary [3]. The well-known general equations of motion and their first integrals are [4]

$$
\begin{aligned}
\frac{\mathrm{d} p}{\mathrm{~d} t}+A_{1} q r= & M g A^{-1}\left(y_{0} \gamma^{\prime \prime}-z_{0} \gamma^{\prime}\right), \\
\frac{\mathrm{d} \gamma}{\mathrm{d} t}=r \gamma^{\prime}-q \gamma^{\prime \prime}, & \\
& \left(p q r, A_{1} B_{1} C_{1}, A B C, \gamma \gamma^{\prime} \gamma^{\prime \prime}, x_{o} y_{0} z_{o}\right), \\
A p^{2}+B q^{2}+C r^{2}-2 M g\left(x_{0} \gamma+y_{0} \gamma^{\prime}+z_{0} \gamma^{\prime \prime}\right)= & \text { const., } \\
(A p) \gamma+(B q) \gamma^{\prime}+(C r) \gamma^{\prime \prime} & =\text { const., } \\
\gamma^{2}+\gamma^{\prime 2}+\gamma^{\prime \prime 2} & =1,
\end{aligned}
$$


where

$$
\begin{aligned}
& A_{1}=\frac{C-B}{A}, \\
& B_{1}=\frac{A-C}{B}, \\
& C_{1}=\frac{B-A}{C} .
\end{aligned}
$$

System (1) of equations of motion represents nonlinear differential equations of the considered problem. These equations are of the first order in unknown angular velocity components $p, q$, and $r$ and geometric angles $\gamma, \gamma^{\prime}$, and $\gamma^{\prime \prime}$. The quantities $A, B$, and $C$ represent the moments of inertia of the body and $\left(x_{0}, y_{0}, z_{0}\right)$ represent its gravity center. $g$ denotes the gravity acceleration. $t$ denotes the time of the motion. The aim is to find the solution to this system using the large parameter method [5].

Let the initial value of the angular velocity component $r=r_{o}$ about the moving $z$ axis be sufficiently small. The following variables are introduced:

$$
\begin{aligned}
& p_{1}=p c^{-1} \sqrt{\gamma_{0}^{\prime \prime},} \\
& q_{1}=q c^{-1} \sqrt{\gamma_{0}^{\prime \prime},} \\
& r_{1}=\frac{r}{r_{0}}, \\
& \gamma_{1}=\frac{\gamma}{\gamma_{0}^{\prime \prime}}, \\
& \gamma_{1}^{\prime}=\frac{\gamma^{\prime}}{\gamma_{0}^{\prime \prime}}, \\
& \gamma_{1}^{\prime \prime}=\frac{\gamma^{\prime \prime}}{\gamma_{0}^{\prime \prime}}, \\
& \tau=r_{o}^{-1} t,
\end{aligned}
$$

$$
\begin{aligned}
& \left(. \equiv \frac{\mathrm{d}}{\mathrm{d} \tau}\right), \\
p_{2}= & p_{1}-e \lambda^{-1}-\lambda^{-1} e_{1} \gamma_{2}, \\
\gamma_{2} & =\gamma_{1}-\lambda^{-1} v p_{2}, \\
c & =\sqrt{\frac{M g \ell}{C}} \\
\lambda & =\frac{c \sqrt{\gamma_{o}^{\prime \prime}}}{r_{0}} \\
e & =\frac{x_{o}^{\prime} A_{1}}{b \omega^{2}} \\
e_{1} & =\frac{z_{o}^{\prime}\left[A_{1} / b-a^{-1}\right]}{\left(1-\omega^{2}\right)} \\
v & =\frac{\left(1+B_{1}\right)}{\left(1-\omega^{2}\right)} \\
\omega^{2} & =-A_{1} B_{1}, \\
a & =\frac{A}{C} \\
b & =\frac{B}{C} \\
x_{o} & =\ell x_{o}^{\prime}, \\
y_{o} & =\ell y_{o}^{\prime}, \\
z_{o}= & \ell z_{o}^{\prime}, \\
\ell^{2} & =x_{o}^{2}+y_{o}^{2}+z_{o}^{2}, \\
& \\
&
\end{aligned}
$$

where $r_{0}$ and $\gamma_{0}^{\prime \prime}$ are the initial values of the corresponding quantities.

The nonlinear equations of motions and their first integrals (1) are reduced to a quasilinear autonomous system [6]:

$$
\begin{aligned}
\ddot{p}_{2}+\omega^{2} p_{2} & =\lambda^{-2} F\left(p_{2}, \dot{p}_{2}, \gamma_{2}, \dot{\gamma}_{2}, \frac{1}{\lambda}\right), \\
\ddot{\gamma}_{2}+\gamma_{2} & =\lambda^{-2} \Phi\left(p_{2}, \dot{p}_{2}, \gamma_{2}, \dot{\gamma}_{2}, \frac{1}{\lambda}\right),
\end{aligned}
$$

where

$$
\begin{aligned}
F= & C_{1} A_{1}^{-1} p_{2} \dot{p}_{2}^{2}+x_{0}^{\prime} \dot{p}_{2} \dot{\gamma}_{2}-y_{0}^{\prime} a^{-1} p_{2} \dot{\gamma}_{2}-y_{0}^{\prime} A_{1}^{-1}\left(A_{1}+a^{-1}\right) \gamma_{2} \dot{p}_{2}-z_{0}^{\prime} a^{-1} p_{2} \\
& -v e_{1}\left(1-\omega^{2}\right) p_{2}-\omega^{2} p_{2} s_{11}+A_{1} b^{-1} x_{0}^{\prime} s_{21}+O\left(\frac{1}{\lambda}\right)+\ldots, \\
\Phi= & -\left(1-C_{1}\right) A_{1}^{-1} p_{2} \dot{p}_{2} \dot{\gamma}_{2}+x_{0}^{\prime} \dot{\gamma}_{2}^{2}-y_{0}^{\prime} \gamma_{2} \dot{\gamma}_{2}-z_{0}^{\prime} b^{-1} \gamma_{2}+x_{0}^{\prime} b^{-1}-A_{1}^{-2} \gamma_{2} \dot{p}_{2}^{2} \\
& +v\left(1-\omega^{2}\right)\left(e+e_{1} \gamma_{2}\right)-\gamma_{2} s_{11}+\left(1+B_{1}\right) p_{2} s_{21}+O\left(\frac{1}{\lambda}\right)+\ldots, \\
s_{11}= & \frac{a\left(p_{20}^{2}-p_{2}^{2}\right)+b\left(\dot{p}_{20}^{2}-\dot{p}_{2}^{2}\right)}{A_{1}^{2}-2\left[x_{0}^{\prime}\left(\gamma_{20}-\gamma_{2}\right)+y_{0}^{\prime}\left(\dot{\gamma}_{20}-\dot{\gamma}_{2}\right)\right]}, \\
s_{21}= & a\left(p_{20} \gamma_{20}-p_{2} \gamma_{2}\right)-b A_{1}^{-1}\left(\dot{p}_{20} \dot{\gamma}_{20}-\dot{p}_{2} \dot{\gamma}_{2}\right), \\
v_{2}= & v-A_{1}^{-1},
\end{aligned}
$$


such that $p_{20}$ and $\gamma_{20}$ are the initial values of the corresponding quantities.

The variables $q_{1}, r_{1}, \gamma_{1}^{\prime}$, and $\gamma_{1}^{\prime \prime}$ are obtained as follows:

$$
\begin{aligned}
q_{1} & =-A_{1}^{-1} \dot{p}_{2}+\lambda^{-1} A_{1}^{-1}\left(y_{0}^{\prime} a^{-1}-e_{2} \dot{\gamma}_{2}\right)+\ldots, \\
r_{1} & =1+0.5 \lambda^{-2} s_{11}+\ldots \\
\gamma_{1}^{\prime} & =\dot{\gamma}_{2}+\lambda^{-1} v_{2} \dot{p}_{2}+\ldots \\
\gamma_{1}^{\prime \prime} & =1+\lambda^{-1} s_{21}+\lambda^{-2}\left(s_{22}-0.5 s_{11}\right)+\ldots
\end{aligned}
$$

where

$$
\begin{aligned}
s_{22}= & a\left[\nu\left(p_{20}^{2}-p_{2}^{2}\right)+e\left(\gamma_{20}-\gamma_{2}\right)+e_{1}\left(\gamma_{20}^{2}-\gamma_{2}^{2}\right)\right] \\
& +b A_{1}^{-1}\left[-\gamma_{2}\left(\dot{p}_{20}^{2}-\dot{p}_{2}^{2}\right)+a^{-1} y_{0}^{\prime}\left(\dot{\gamma}_{20}-\dot{\gamma}_{2}\right)-e_{2}\left(\dot{\gamma}_{20}^{2}-\dot{\gamma}_{2}^{2}\right)\right], . \\
e_{2}= & e_{1}+a^{-1} z_{o}^{\prime}
\end{aligned}
$$

Assuming that the velocity $r_{o}$ is sufficiently small, the parameter $\lambda$ is large.

\section{Construction of Periodic Solutions, with Zero Basic Amplitudes}

In this section, the periodic solutions, with zero basic amplitudes [7], of the autonomous system (4) are achieved and the large parameter method is applied. Without loss of generality of solutions, it is considered that

$$
p_{2}(0,0)=\dot{p}_{2}(0,0)=\dot{\gamma}_{2}\left(0, \frac{1}{\lambda}\right)=0 \text {. }
$$

Consider the generating system $((1 / \lambda)=0)$, that is, $(\lambda \longrightarrow \infty)$, of $(4)$ in the form:

$$
\begin{aligned}
\ddot{p}_{2}^{(0)}+\omega^{2} p_{2}^{(0)} & =0, \\
\ddot{\gamma}_{2}^{(0)}+\gamma_{2}^{(0)} & =0,
\end{aligned}
$$

with a period $T_{0}=2 \pi n$. There are three possibilities of the values of frequency $\omega$ which are $1-\omega=1 ; 2-\omega=m / n$ where $m$ and $n$ are primes; $3-\omega$ equals an irrational number.

Consider the case when $\omega=m / n$, then the solution of the generating system (9) becomes

$$
\begin{aligned}
& p_{2}^{(0)}=a_{0}^{*} \cos \omega \tau, \\
& \gamma_{2}^{(0)}=b_{0}^{*} \cos \tau,
\end{aligned}
$$

where $a_{0}^{*}$ and $b_{0}^{*}$ are the constants to be determined. The autonomous system (4) has periodic solutions with a period $T_{0}+\alpha$, where $\alpha$ is a function of $1 / \lambda$ such that $\alpha(0)=0$. These solutions are reduced to the generating ones (10) when $(1 / \lambda)=0(\lambda \longrightarrow \infty)$ and written in the form:

$$
\begin{aligned}
& p_{2}=a^{*} \cos \psi+\sum_{n=1}^{N}\left(\frac{1}{\lambda}\right)^{n} p_{n}^{*}\left(a^{*}, \psi\right)+O\left(\frac{1}{\lambda}\right)^{N+1}, \\
& \gamma_{2}=b^{*} \cos \phi+\sum_{n=1}^{N}\left(\frac{1}{\lambda}\right)^{n} \gamma_{n}^{*}\left(a^{*}, \phi\right)+O\left(\frac{1}{\lambda}\right)^{N+1} .
\end{aligned}
$$

With initial conditions:

$$
\begin{aligned}
& p_{2}\left(0, \frac{1}{\lambda}\right)=a^{*}=a_{0}^{*}+a^{*}\left(\frac{1}{\lambda}\right), \\
& \gamma_{2}\left(0, \frac{1}{\lambda}\right)=b^{*}=b_{0}^{*}+b^{*}\left(\frac{1}{\lambda}\right), \\
& \dot{\gamma}_{2}\left(0, \frac{1}{\lambda}\right)=0,
\end{aligned}
$$

where $a^{*}(1 / \lambda)=0$ and $b^{*}(1 / \lambda)=0$ when $(1 / \lambda)=0$.

From the first integral (4) and initial conditions (12), one has the following:

$$
\begin{aligned}
& 0<b_{0}^{*}=\left(1-\gamma_{0}^{\prime \prime 2}\right)^{1 / 2}\left(\gamma_{0}^{\prime \prime}\right)^{-1}<\infty, \\
& b^{*}\left(\frac{1}{\lambda}\right)=-\left(\frac{1}{\lambda}\right) \nu\left[a_{0}^{*}+a^{*}\left(\frac{1}{\lambda}\right)\right]+\ldots
\end{aligned}
$$

Let $a^{*}, \psi$, and $\phi$ change with time according to

$$
\begin{aligned}
& \dot{a}^{*}=\sum_{n=1}^{N}\left(\frac{1}{\lambda}\right)^{n} A_{n}^{*}\left(a^{*}\right)+O\left(\frac{1}{\lambda}\right)^{N+1}, \\
& \dot{\psi}=\omega+\sum_{n=1}^{N}\left(\frac{1}{\lambda}\right)^{n} \psi_{n}\left(a^{*}\right)+O\left(\frac{1}{\lambda}\right)^{N+1}, \\
& \dot{\phi}=1+\sum_{n=1}^{N}\left(\frac{1}{\lambda}\right)^{n} \phi_{n}\left(a^{*}\right)+O\left(\frac{1}{\lambda}\right)^{N+1} .
\end{aligned}
$$

The following derivatives are obtained: 


$$
\begin{aligned}
\dot{p}_{2}= & -a^{*} \omega \sin \psi+O\left(\frac{1}{\lambda}\right), \\
\dot{\gamma}_{2}= & -b^{*} \sin \phi+O\left(\frac{1}{\lambda}\right), \\
\ddot{p}_{2}= & -a^{*} \omega^{2} \cos \psi+\frac{1}{\lambda}\left[\omega^{2} \frac{\partial^{2} p_{1}^{*}}{\partial \psi^{2}}-2 a^{*} \omega \psi_{1} \cos \psi-2 \omega A_{1}^{*} \sin \psi\right] \\
& +\left(\frac{1}{\lambda}\right)^{2}\left[2 \omega A_{1}^{*} \frac{\partial^{2} p_{1}^{*}}{\partial a^{*} \partial \psi}-2\left(\omega A_{2}^{*}+A_{1}^{*} \psi_{1}\right) \sin \psi+A_{1}^{*} \frac{d A_{1}^{*}}{d a^{*}} \cos \psi+\omega^{2} \frac{\partial^{2} p_{2}^{*}}{\partial \psi^{2}}\right. \\
& \left.+2 \omega \psi_{1} \frac{\partial^{2} p_{1}^{*}}{\partial \psi^{2}}-a^{*}\left(\psi_{1}^{2}+2 \omega \psi_{2}\right) \cos \psi-a^{*} A_{1}^{*} \sin \psi \frac{d \psi_{1}}{d a^{*}}\right]+O\left(\frac{1}{\lambda}\right)^{3}, \\
\ddot{\gamma}_{2}= & -b^{*} \cos \phi+\frac{1}{\lambda}\left[\frac{\partial^{2} \gamma_{1}^{*}}{\partial \phi^{2}}-2 b^{*} \phi_{1} \cos \phi\right]+\left(\frac{1}{\lambda}\right)^{2}\left[\frac{\partial^{2} \gamma_{2}^{*}}{\partial \phi^{2}}+2 \phi_{1} \frac{\partial^{2} \gamma_{1}^{*}}{\partial \phi^{2}}\right. \\
& \left.-b^{*}\left(\phi_{1}^{2}+2 \phi_{2}\right) \cos \phi+2 A_{1}^{*} \frac{\partial^{2} \gamma_{1}^{*}}{\partial a^{*} \partial \phi}-b^{*} A_{1}^{*} \frac{d \phi_{1}}{d a^{*}} \sin \phi\right]+O\left(\frac{1}{\lambda}\right)^{3} .
\end{aligned}
$$

From (5), (7), (11), and (17), it is obtained that

$$
\begin{aligned}
s_{11}^{(0)}= & a a_{0}^{* 2}\left(\cos ^{2} \psi_{0}-\cos ^{2} \psi\right)-b A_{1}^{-2} a_{0}^{* 2} \omega^{2} \sin ^{2} \psi-2 b_{0}^{*}\left[x_{0}^{\prime}\left(\cos \phi_{0}-\cos \phi\right)+y_{0}^{\prime} \sin \phi\right], \\
s_{21}^{(0)}= & a_{0}^{*} b_{0}^{*}\left[a\left(\cos \psi_{0} \cos \phi_{0}-\cos \psi \cos \phi\right)+b A_{1}^{-1} \omega \sin \psi \sin \phi\right], \\
s_{22}^{(0)}= & a\left[v a_{0}^{* 2}\left(\cos ^{2} \psi_{0}-\cos ^{2} \psi\right)+e b_{0}^{*}\left(\cos \phi_{0}-\cos \phi\right)+e_{1} b_{0}^{* 2}\left(\cos ^{2} \phi_{0}-\cos ^{2} \phi\right)\right] \\
& +b A_{1}^{-1}\left[v_{2} a_{0}^{* 2} \omega^{2} \sin ^{2} \psi+a^{-1} y_{0}^{\prime} b_{0}^{*} \sin \phi+e_{2} b_{0}^{* 2} \sin ^{2} \phi\right],
\end{aligned}
$$

where $\psi_{0}$ and $\phi_{0}$ are the initial values of the corresponding quantities.

Using (5), (11), and (17), the following is obtained:

$$
\begin{aligned}
F^{(0)}= & C_{1} A_{1}^{-1} a_{0}^{* 3} \omega^{2} \cos \psi \sin ^{2} \psi+\omega a_{0}^{*} b_{0}^{*} x_{0}^{\prime} \sin \psi \sin \phi \\
& +a^{-1} a_{0}^{*} b_{0}^{*} y_{0}^{\prime} \cos \psi \sin \phi+\omega A_{1}^{-1}\left(A_{1}+a^{-1}\right) a_{0}^{*} b_{0}^{*} y_{0}^{\prime} \sin \psi \cos \phi \\
& -z_{0}^{\prime} a^{-1} a_{0}^{*} \cos \psi-v e_{1}\left(1-\omega^{2}\right) a_{0}^{*} \cos \psi \\
& -\omega^{2} a_{0}^{*} \cos \psi\left\{a a_{0}^{* 2}\left(\cos ^{2} \psi_{0}-\cos ^{2} \psi\right)-b A_{1}^{-2} a_{0}^{* 2} \omega^{2} \sin ^{2} \psi\right. \\
& \left.-2 b_{0}^{*}\left[x_{0}^{\prime}\left(\cos \phi_{0}-\cos \phi\right)+y_{0}^{\prime} \sin \phi\right]\right\} \\
& +A_{1} b^{-1} x_{0}^{\prime} a_{0}^{*} b_{0}^{*}\left[a\left(\cos \psi_{0} \cos \phi_{0}-\cos \psi \cos \phi\right)+b A_{1}^{-1} \omega \sin \psi \sin \phi\right] \\
\Phi^{(0)}= & \frac{1}{2}\left(C_{1}-1\right) A_{1}^{-1} \omega a_{0}^{* 2} b_{0}^{*} \sin 2 \psi \sin \phi+\frac{1}{2} x_{0}^{\prime} b_{0}^{* 2}(1-\cos 2 \phi) \\
& +\frac{1}{2} y_{0}^{\prime} b_{0}^{* 2} \sin 2 \phi-z_{0}^{\prime} b^{-1} b_{0}^{*} \cos \phi+x_{0}^{\prime} b^{-1} \\
& -\frac{1}{2} A_{1}^{-2} \omega^{2} a_{0}^{* 2} b_{0}^{*}(1-\cos 2 \psi) \cos \phi+v e\left(1-\omega^{2}\right)+v e_{1}\left(1-\omega^{2}\right) b_{0}^{*} \cos \phi
\end{aligned}
$$


Advances in Astronomy

5

$$
\begin{aligned}
& -a a_{0}^{* 2} b_{0}^{*} \cos ^{2} \psi_{0} \cos \phi+\frac{1}{2} a a_{0}^{* 2} b_{0}^{*}(1+\cos 2 \psi) \cos \phi \\
& +\frac{1}{2} b A_{1}^{-2} \omega^{2} a_{0}^{* 2} b_{0}^{*}(1-\cos 2 \psi) \cos \phi+2 x_{0}^{\prime} b_{0}^{* 2} \cos \phi_{0} \cos \phi \\
& -x_{0}^{\prime} b_{0}^{* 2}(1+\cos 2 \phi)+y_{0}^{\prime} b_{0}^{* 2} \sin 2 \phi+a_{0}^{* 2} b_{0}^{*}\left(1+B_{1}\right)\left[b A_{1}^{-1} \omega \sin \psi \sin \phi\right. \\
& \left.+a\left(\cos \psi_{0} \cos \phi_{0}-\cos \psi \cos \phi\right)\right] \cos \psi
\end{aligned}
$$

Substituting (11), (17), and (19) into system (4) and equating coefficients of similar power terms of $1 / \lambda$, the following is obtained:

$$
\begin{aligned}
& \frac{\partial^{2} p_{1}^{*}}{\partial \psi^{2}}+p_{1}^{*}=\frac{2 a_{0}^{*}}{\omega} \psi_{1} \cos \psi+\frac{2 A_{1}^{*}}{\omega} \sin \psi \\
& \frac{\partial^{2} \gamma_{1}^{*}}{\partial \phi^{2}}+\gamma_{1}^{*}=2 b_{0}^{*} \phi_{1} \cos \phi \\
& \frac{\partial^{2} p_{2}^{*}}{\partial \psi^{2}}+p_{2}^{*}=\frac{2 A_{2}^{*}}{\omega} \sin \psi+\frac{a_{0}^{*}}{\omega^{2}}\left[2 \omega \psi_{2}+\frac{1}{4} \omega^{2} C_{1} A_{1}^{-1} a_{0}^{* 2}+\frac{3}{4} \omega^{2} a a_{0}^{* 2}-a a_{0}^{* 2}\right. \\
& \left.-z_{0}^{\prime} a^{-1}-v e_{1}\left(1-\omega^{2}\right)+\frac{1}{4} b A_{1}^{-2} a_{0}^{* 2} \omega^{4}+2 \omega^{2} x_{0}^{\prime} b_{0}^{*} \cos \phi_{0}\right] \cos \psi \\
& +\frac{1}{4} a_{0}^{* 3}\left(a-C_{1} A_{1}^{-1}-\omega^{2} b A_{1}^{-2}\right) \cos 3 \psi+\frac{a a_{0}^{*}}{\omega^{2}} x_{0}^{\prime} A_{1} b^{-1} b_{0}^{*} \cos \psi_{0} \cos \phi_{0} \\
& +x_{0}^{\prime} a_{0}^{*} b_{0}^{*}\left(\frac{1}{\omega}-\frac{a A_{1}}{2 b \omega^{2}}-1\right) \cos (\phi-\psi)-x_{0}^{\prime} a_{0}^{*} b_{0}^{*}\left(1+\frac{1}{\omega}+\frac{a A_{1}}{2 b \omega^{2}}\right) \cos (\phi+\psi) \\
& +y_{0}^{\prime} a_{0}^{*} b_{0}^{*}\left(1+\frac{1}{2 a \omega^{2}}-\frac{A_{1}+a^{-1}}{2 A_{1} \omega}\right) \sin (\phi-\psi)+y_{0}^{\prime} a_{0}^{*} b_{0}^{*}\left(1+\frac{1}{2 a \omega^{2}}+\frac{A_{1}+a^{-1}}{2 A_{1} \omega}\right) \sin (\phi+\psi), \\
& \frac{\partial^{2} \gamma_{2}^{*}}{\partial \phi^{2}}+\gamma_{2}^{*}=\left[2 \phi_{2}-z_{0}^{\prime} b^{-1}+\frac{1}{2} A_{1}^{-2} \omega^{2} a_{0}^{* 2}(b-1)+v e_{1}\left(1-\omega^{2}\right)-a a_{0}^{* 2} \cos ^{2} \psi_{0}\right. \\
& \left.-\frac{1}{2} a B_{1} a_{0}^{* 2}+2 x_{0}^{\prime} b_{0}^{*} \cos \phi_{0}\right] b_{0}^{*} \cos \phi-\frac{1}{2} x_{0}^{\prime} b_{0}^{* 2}+x_{0}^{\prime} b^{-1}+\nu e\left(1-\omega^{2}\right) \\
& +\left(1+B_{1}\right) a a_{0}^{* 2} b_{0}^{*} \cos \psi_{0} \cos \phi_{0} \cos \psi-\frac{3}{2} x_{0}^{\prime} b_{0}^{* 2} \cos 2 \phi+\frac{3}{2} y_{0}^{\prime} b_{0}^{* 2} \sin 2 \phi \\
& +a_{0}^{* 2}\left\{\left[\frac{1}{2} A_{1}^{-2} \omega^{2}(1-b)-\frac{1}{2} a B_{1}+A_{1}^{-1} \omega b_{0}^{*}(b-1)\right] \cos (2 \psi-\phi)\right. \\
& \left.+\left[\frac{1}{2} A_{1}^{-2} \omega^{2}(1-b)-\frac{1}{2} a B_{1}-A_{1}^{-1} \omega b_{0}^{*}(b-1)\right] \cos (2 \psi+\phi)\right\} .
\end{aligned}
$$

Canceling the singular terms [8] from (20), one gets 


$$
\begin{aligned}
\psi_{1}= & A_{1}^{*}=\phi_{1}=A_{2}^{*}=0, \\
\psi_{2}= & \frac{1}{2 \omega}\left[-\frac{1}{4} \omega^{2} C_{1} A_{1}^{-1} a_{0}^{* 2}-\frac{3}{4} \omega^{2} a a_{0}^{* 2}+a a_{0}^{* 2}+z_{0}^{\prime} a^{-1}+v e_{1}\left(1-\omega^{2}\right)\right. \\
& \left.-\frac{1}{4} b A_{1}^{-2} \omega^{4} a_{0}^{* 2}-2 \omega^{2} x_{0}^{\prime} b_{0}^{*} \cos \phi_{0}\right], \\
\phi_{2}= & \frac{1}{2}\left[z_{0}^{\prime} b^{-1}-\frac{1}{2} A_{1}^{-2} \omega^{2} a_{0}^{* 2}(b-1)-v e_{1}\left(1-\omega^{2}\right)+a a_{0}^{* 2}\left(\frac{1}{2} B_{1}+\cos ^{2} \psi_{0}\right)\right. \\
& \left.-2 x_{0}^{\prime} b_{0}^{*} \cos \phi_{0}\right] .
\end{aligned}
$$

Substituting (21) into (14), (15), and (16) and integrating, it is obtained that

$$
\begin{aligned}
a^{*}= & a_{0}^{*} \text { (arbitrary const.), } \\
\psi= & \omega \tau+\frac{1}{2}\left(\frac{1}{\lambda}\right)^{2}\left[-\frac{1}{4} \omega C_{1} A_{1}^{-1} a_{0}^{* 2}-\frac{3}{4} \omega a a_{0}^{* 2}+a a_{0}^{* 2} \omega^{-1}+z_{0}^{1} a^{-1} \omega^{-1}\right. \\
& \left.+\nu e_{1}\left(\omega^{-1}-\omega\right)-\frac{1}{4} b A_{1}^{-2} \omega^{3} a_{0}^{* 2}-2 \omega x_{0}^{\prime} b_{0}^{*} \cos \phi_{0}\right] \tau, \\
\phi= & \tau+\frac{1}{2}\left(\frac{1}{\lambda}\right)^{2}\left[z_{0}^{\prime} b^{-1}-\frac{1}{2} A_{1}^{-2} \omega^{2} a_{0}^{* 2}(b-1)-v e_{1}\left(1-\omega^{2}\right)+a a_{0}^{* 2}\left(1+\frac{1}{2} B_{1}\right)-2 x_{0}^{\prime} b_{0}^{*}\right] \tau .
\end{aligned}
$$

From the previous results, the following is obtained:

$$
\begin{aligned}
& \psi(0)=\psi_{0}=0, \\
& \phi(0)=\phi_{0}=0 .
\end{aligned}
$$

Making use of (21), (22), (11), and (13), the periodic solutions $p_{2}$ and $\gamma_{2}$ of the autonomous system are deduced. Using (6), (18), (22), and (23), the following periodic solutions, with zero basic amplitudes, are obtained:

$$
\begin{aligned}
p_{1}= & -\frac{x_{0}^{\prime}}{\lambda b B_{1}}+\frac{1}{\lambda} e_{1} b_{0}^{*} \cos \tau+\ldots, \\
q_{1}= & \frac{y_{0}^{\prime}}{\lambda a A_{1}}+\frac{1}{\lambda} e_{1} A_{1}^{-1} b_{0}^{*} \sin \tau+\ldots, \\
r_{1}= & 1-\frac{1}{\lambda^{2}} b_{0}^{*}\left[x_{0}^{\prime}(1-\cos \tau)+y_{0}^{\prime} \sin \tau\right]+\ldots, \\
\gamma_{1}= & b_{0}^{*} \cos \tau+\ldots, \\
\gamma_{1}^{\prime}= & -b_{0}^{*} \sin \tau+\ldots, \\
\gamma_{1}^{\prime \prime}= & 1+\frac{1}{\lambda^{2}}\left[b_{0}^{*}(1-a)^{-1} x_{0}^{\prime}+\frac{1}{2} b_{0}^{* 2} z_{0}^{\prime}\left(\frac{a-b}{a+b-1}\right)+b_{0}^{*}(1-b)^{-1} y_{0}^{\prime} \sin \tau\right. \\
& \left.-b_{0}^{*}(1-a)^{-1} x_{0}^{\prime} \cos \tau-\frac{1}{2} b_{0}^{* 2} z_{0}^{\prime}\left(\frac{a-b}{a+b-1}\right) \cos 2 \tau\right]+\ldots, \\
\text { the period } \alpha \text { becomes } & \alpha\left(\frac{1}{\lambda}\right)=\frac{2}{\lambda} \pi n\left[b_{0}^{*} x_{0}^{\prime}-z_{0}^{\prime}\right]+\ldots
\end{aligned}
$$

where the correction of the period $\alpha$ becomes 


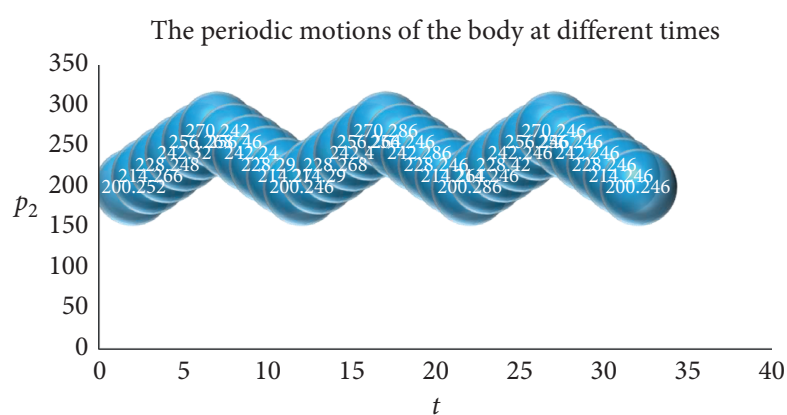

Figure 1: The periodic motion $p_{2}$ against the time $t$.

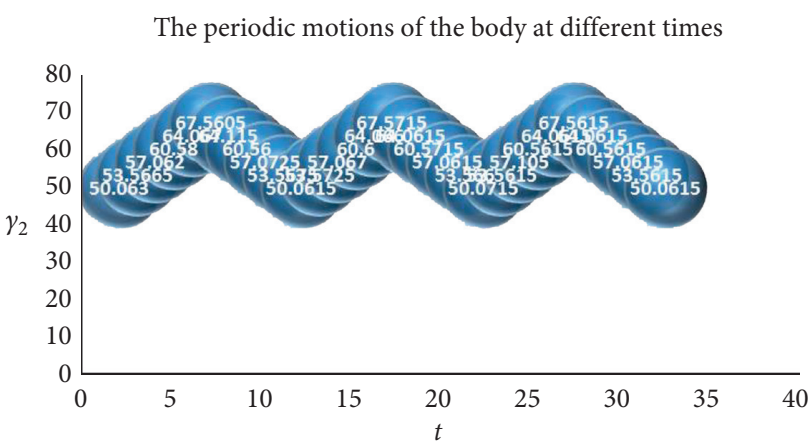

FIgURE 2: The periodic motion $\gamma_{2}$ against the time $t$.

The stability of the solutions and the amplitude of the motion

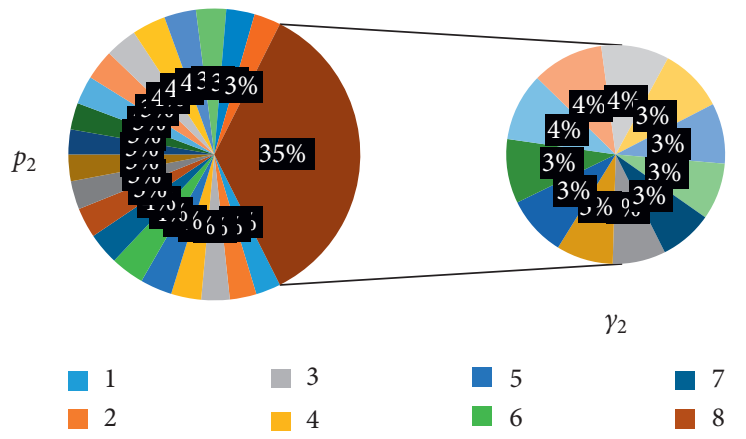

Figure 3: The stability of the solution $p_{2}$ against $\gamma_{2}$.

\section{Conclusion}

It is concluded that the method of the small parameter failed to solve this problem under the studied condition $r_{0}$ which is sufficiently small because achieving the solutions by this method depends on assuming sufficiently large angular velocity $r_{0}$ to define the small parameter $(\varepsilon)$ proportional to $\left(1 / r_{o}\right)$. With the sufficiently small assumption, the choosing of the small parameter $(\varepsilon)$ is impossible, and so the author had to look for another technique.

The large parameter technique is the only one that solves this problem under the studied condition. The advantage of this method is that you save an enormous amount of energy given to the body at the start of the motion. The presented method proves the ability to solve this problem when the component of the angular velocity about the moving $z$-axis is sufficiently small. Under this technique, gyroscopic motions are obtained under low energy initially instead of high energy in using the small parameter technique. It is clear about the periodicity of the solutions $p_{2}$ and $\gamma_{2}$ from Figures 1 and 2 in a defined interval of time. The simple smooth closed curves with different amplitudes of the solution $p_{2}$ against $\gamma_{2}$ show the stability [9] of the motions, see Figure 3 .

\section{Data Availability}

No datasets were generated or analyzed during the current study.

\section{Conflicts of Interest}

The author declares that there are no conflicts of interest.

\section{References}

[1] F. L. Chernousko, L. D. Akulenko, and D. D. Leshchenko, Evolution of Motions of a Rigid Body about its Center of Mass, p. 1, Springer International Publishing AG, Cham, Switzerland, 2017.

[2] G. M. Scarpello and D. Ritelli, "Motions about a fixed point by hypergeometric functions: new non-complex analytical solutions and integration of the herpolhode," Celestial Mechanics and Dynamical Astronomy, vol. 130, no. 42, 2018.

[3] T. S. Amer and W. S. Amer, "Substantial condition for the fourth first integral of the rigid body problem," Mathematics and Mechanics of Solids, vol. 23, no. 8, pp. 1237-1246, 2018.

[4] A. V. Borisov and I. S. Mamaev, Rigid Body Dynamics, Higher education press and Walter de Gruyter GmbH, Berlin, Germany, 2018.

[5] A. H. Nayfeh, Introduction to Perturbation Techniques, pp. 360-364, WILEY-VCH Verlag GmbH \& Co. KGaA, Weinheim, Germany, 2011.

[6] A. I. Ismail and T. S. Amer, "The fast spinning motion of a rigid body in the presence of a gyrostatic momentum," Acta Mechanica, vol. 154, no. 1-4, pp. 31-46, 2002.

[7] https://courses.lumenlearning.com/physics/chapter/16-10superposition-and-interference/.

[8] T. D. Lee, Selected Papers: Field Theory and Symmetry Principles, Contemporary physicists Gerald Feinberg, Birkhäuser, Basel, Switzerland, 1986.

[9] http://ddebiftool.sourceforge.net/demos/neuron/html/demo 1_psol.html. 